\title{
Enhancement of damage indicators in wavelet and curvature analysis
}

\author{
B K RAGHU PRASAD ${ }^{1}$, N LAKSHMANAN ${ }^{2}$, K MUTHUMANI $^{2}$ \\ and N GOPALAKRISHNAN ${ }^{2}$
}

${ }^{1}$ Dept. of Civil Engineering, Indian Institute of Science, Bangalore 560 012, India

${ }^{2}$ Structural Engineering Research Centre, Chennai, India

e-mail: bkr@civil.iisc.ernet.in

\begin{abstract}
Damage in a structural element induces a small perturbation in its static or dynamic displacement profile which can be captured by wavelet analysis. The paper presents the wavelet analysis of damaged linear structural elements using DB4 or BIOR6. 8 family of wavelets. An expression is developed for computing the natural frequencies of a damaged beam using first order perturbation theory. Starting with a localized reduction of $E I$ at the mid-span of a simply supported beam, damage modelling is done for a typical steel beam element. Wavelet analysis is performed for this damage model for displacement, rotation and curvature mode shapes as well as static displacement profiles. Damage indicators like displacement, slope and curvature are magnified under higher modes. Instantaneous step-wise linearity is assumed for all the nonlinear elements. A localization scheme with arbitrararily located curvature nodes within a pseudo span is developed for steady state dynamic loads, such that curvature response and damages are maximized and the scheme is numerically tested and proved.
\end{abstract}

Keywords. Wavelet transforms; damage identification; modal parameters; enhanced damage indicators.

\section{Introduction}

Research in the area of damage detection and identification saw a quantum jump during the late eighties and early nineties (Lakshmanan et al 1991; Rajagopalan et al 1996, 1999; Hassiotis et al 1993; Hassiotis 2000). Natural frequency is a less sensitive parameter to structural damage. If it is assumed that $(E I)_{\text {eff }}$ undergoes a global change ' $\alpha$ ', then change in the natural frequency could be approximated as $0 \cdot 5 \alpha$. Also, if a quadratic relationship is assumed between the strength and modulus, as typical of concrete, with E proportional to $\sqrt{f_{c k}}$, natural frequency is proportional to $f_{c k}^{0 \cdot 25}$. This implies that if a $20 \%$ drop occurs in the strength of

A list of symbols is given at the end of the paper.

This paper is dedicated to Prof R N Iyengar of the Indian Institute of Science on the occasion of his formal retirement. 
a system, its natural frequency drops by $5 \%$. On the contrary, increase in static deflection is directly proportional to the decrease in $(E I)_{\text {eff }}$. All these counter arguments notwithstanding, damage identification is still pursued through dynamic measurement techniques by engineers and scientists, due to the following reasons.

(a) Progress made in digital electronics has propelled an enormous spurt in dynamic measurement techniques involving smart, laser-based and MEMS sensors, data acquisition and wireless communications. The technology of transducers and the connected integrated circuits and amplifiers, has seen enormous miniaturization, capacity increase, coupled with sharp fall in their prices. Sensitivities of the instruments have increased by orders of magnitude and even a small force is sufficient to excite a structure at its natural frequencies.

(b) Whereas an elaborate amount of instrumentation and mounting system is required to evaluate a structure under in-situ conditions with static loading, virtually zero paraphernalia is required for dynamic response measurements with ambient excitations.

(c) Techniques like fast Fourier transforms have been embedded into chips to form firmware and these analysers have also been made portable for easy field use. The idea of condition monitoring has crept into civil engineering with periodic collection and Fourier synthesis of vibration signatures being made mandatory for certain bridges.

(d) More than natural frequencies and displacement mode shapes, rotation and curvature mode shapes are better indicators of damage and these coupled with appropriate signal enhancement techniques could be used as ideal damage indicators (Pandey et al 1991; Abdel Wahab et al 1999; Ratcliffe 2000; Abdo et al 2002; Owolabi et al 2003).

(e) Measurement of modal parameters could be used to work back the system characteristics like stiffness, flexibility and mass distribution (Sheinman 1996).

(f) Development of new signal processing techniques like wavelet transforms has entered in a large way in structural engineering as a tool to magnify localized damages in mode shapes and for other applications (Liew et al 1998; Gurley et al 1999; Hou et al 2000; Melhem et al 2003; Hera et al 2004; Kim et al 2004)

(g) Ambient vibration data generated due to the movement of a vehicle on a bridge can be used to identify the system parameters of a bridge, both for linear and nonlinear systems (Majumder \& Manohar 2003, 2004).

(h) Wave-propagation techniques with sonic, ultra-sonic and electro-magnetic waves are other promising damage detection tools.

\section{Sensitivity analysis of eigenvalues and eigenvectors}

Sometimes, it is required to study the changes occurring in measurable output parameters like frequencies and mode shapes, when system characteristics like stiffness and mass undergo small changes. This is done through perturbation analysis of the non-degenerate dynamic system with small perturbations occurring in its stiffness and mass matrices.

The unperturbed (zeroth-order) problem is

$$
K_{0} p_{i}^{(0)}=\lambda_{i}^{(0)} M_{0} p_{i}^{(0)} .
$$

If $K_{1}$ and $M_{1}$ be the small perturbations in stiffness and mass matrices respectively, then the perturbed problem could be written as,

$$
\left(K_{0}+K_{1}\right) p_{i}=\lambda_{i}\left(M_{0}+M_{1}\right) p_{i} .
$$


The eigenvalues and vectors can be expanded in terms of a parameter $\gamma$, such that the zeroth, first etc. powers of $\gamma$ correspond to the zeroth, first, orders of perturbation analysis. The perturbed portion of the original matrix, namely $K_{1}$ and $M_{1}$ can be replaced by $\gamma K_{1}$ and $\gamma M_{1}$ and $\lambda_{i}$ and $p_{i}$ expressed as power series in $\gamma$.

The perturbed eigenvalues and vectors are written as,

$$
\begin{aligned}
& \lambda_{i}=\lambda_{i}^{(0)}+\gamma \cdot \lambda_{i}^{(1)}+\gamma^{2} \lambda_{i}^{(2)}+\cdots, \\
& p_{i}=p_{i}^{(0)}+\gamma \cdot p_{i}^{(1)}+\gamma^{2} p_{i}^{(2)}+\cdots
\end{aligned}
$$

Substituting this in the modified eigenvalue problem,

$$
\begin{aligned}
\left(K_{0}+\right. & \left.\gamma \cdot K_{1}\right)\left(p_{i}^{(0)}+\gamma \cdot p_{i}^{(1)}+\gamma^{2} p_{i}^{(2)}+\cdots\right) \\
= & \left(\lambda_{i}^{(0)}+\gamma \cdot \lambda_{i}^{(1)}+\gamma^{2} \lambda_{i}^{(2)}+\cdots\right)\left(M_{0}+\gamma \cdot M_{1}\right) \\
& \times\left(p_{i}^{(0)}+\gamma \cdot p_{i}^{(1)}+\gamma^{2} p_{i}^{(2)}+\cdots\right) .
\end{aligned}
$$

After equating the coefficients of equal powers of $\gamma$ on both sides of the equation, we can write the zeroth, first and higher order perturbation equations. These are,

$$
\begin{aligned}
& K_{0} p_{i}^{(0)}=\lambda_{i}^{(0)} M_{0} p_{i}^{(0)}, \\
& K_{0} p_{i}^{(1)}+K_{1} p_{i}^{(0)}=\lambda_{i}^{(0)} M_{0} p_{i}^{(1)}+\lambda_{i}^{(0)} M_{1} p_{i}^{(0)}+\lambda_{i}^{(1)} M_{0} p_{i}^{(0)} .
\end{aligned}
$$

It is seen that the zeroth order problem is the unperturbed problem. The vector $p_{i}^{(1)}$, can be expanded on the basis of unperturbed eigenvectors and written as,

$$
p_{i}^{(1)}=\sum_{r} c_{i r} p_{r}^{(0)} .
$$

After simplification and imposing ortho-normalizing properties of both old and new eigenvectors, the first-order perturbation calculations of the ith eigenvalues and vectors can be written as

$$
\begin{aligned}
& \lambda_{i}=\lambda_{i}^{(0)}+p_{i}^{(0) T}\left(K_{1}-\lambda_{i}^{(0)} M_{1}\right) p_{i}^{(0)}, \\
& p_{i}=p_{i}^{(0)}-\left(\frac{1}{2} p_{i}^{(0) T} M_{1} p_{i}^{(0)}\right) p_{i}^{(0)}+\sum_{\substack{r=1 \\
r \neq i}}^{n}\left(\frac{p_{r}^{(0) T}\left(K_{1}-\lambda_{i}^{(0)} M_{1}\right) p_{i}^{(0)}}{\lambda_{i}^{(0)}-\lambda_{r}^{(0)}}\right) p_{r}^{(0)} .
\end{aligned}
$$

For the condition of a damaging structure, wherein the mass matrix does not undergo any change from the original matrix, the above equation can be further simplified as,

$$
\begin{aligned}
& \lambda_{i}=\lambda_{i}^{(0)}+p_{i}^{(0) T} K_{1} p_{i}^{(0)}, \\
& p_{i}=p_{i}^{(0)}+\sum_{\substack{r=1 \\
r \neq i}}^{n}\left(p_{r}^{(0) T} K_{1} p_{i}^{(0)} /\left(\lambda_{i}^{(0)}-\lambda_{r}^{(0)}\right)\right) p_{r}^{(0)},
\end{aligned}
$$




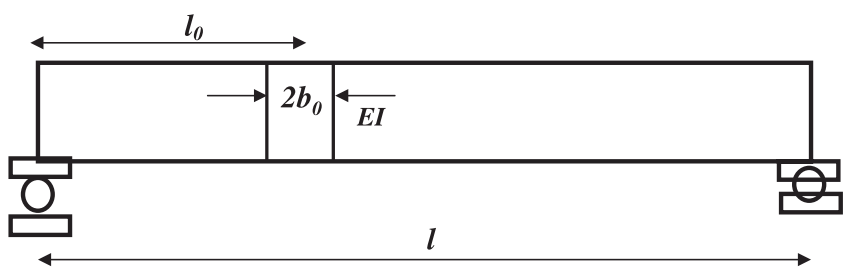

Figure 1. A simply supported beam with reduced $E I$ for a portion of its length.

from the above expressions, it can be noted that only the ith unperturbed parameters enter into the calculations of perturbed eigenvalues, whereas the complete unperturbed eigen solution is required for the computation of perturbed eigevectors.

Using the above perturbation equation the reduced natural frequencies of a damaged simply supported beam is derived (figure 1). Damage is assumed in the form of a reduced $\alpha E I$, situated at a distance of $l_{0}$ from the left end of the beam. The length of the damaged section is assumed as $2 b_{0} . l_{1}$ and $l_{2}$ are the two temporary variables where $l_{0}=\left(l_{1}+l_{2}\right) / 2$ and $2 b_{0}=\left(l_{2}-l_{1}\right)$. The perturbation equation states that the new values of frequencies are obtained using the original un-perturbed Eigen functions.

$$
\left\{\int_{0}^{l_{1}} \frac{1}{2} E I \cdot\left(y^{\prime \prime}\right)^{2} \mathrm{~d} x+\int_{l_{1}}^{l_{2}} \frac{1}{2} \alpha E I \cdot\left(y^{\prime \prime}\right)^{2} \mathrm{~d} x+\int_{l_{2}}^{l} \frac{1}{2} E I \cdot\left(y^{\prime \prime}\right)^{2} \mathrm{~d} x\right\} / \int \frac{1}{2} \bar{m} y^{2} \mathrm{~d} x
$$

where $y(x)=\sin (\pi n x / l)$.

Making a substitution of $\begin{gathered}\beta=1-\alpha \\ 0 \leq \alpha \leq 1\end{gathered}$ in the previous equation and simplifying, the following expression is obtained for the damaged simply supported beam:

$$
\left(\frac{\omega_{d}}{\omega}\right)_{n}=\left\{1-2 \beta\left[\frac{b_{0}}{l}-\frac{1}{2 n \pi}\left(\cos \frac{2 n \pi l_{0}}{l} \cdot \sin \frac{2 n \pi b_{0}}{l}\right)\right]\right\}^{1 / 2} .
$$

In the case of a beam with uniform reduction of $E I$ to $\alpha E I$ for the whole of its length, the equation reduces to the simplest case possible,

$$
\left(\omega_{d} / \omega\right)_{n}=(1-\beta)^{1 / 2}=\sqrt{\alpha} .
$$

If further simplifications are made in the above equation for the case of a crack situated at the centre and for values of $b_{0}$ tending towards zero,

$$
\left(1-\left(\omega_{d} / \omega\right)\right)_{n} \cong\left(\beta b_{0} / l\right)\left[1-(-1)^{n}\right] .
$$

The above equation states that for a centrally situated crack, even-numbered modes have no reduction in their frequencies, which is predictable. Also, the rate of fall of frequency is linearly proportional to the width of damaged zone.

A computational exercise is carried out for the verification of the above equation such that for a simply supported reinforced concrete beam, the loading is such that $10 \%$ of the lengths near both supports remain uncracked, whereas the middle portion is cracked, such that effective $E I$ is $50 \%$ of the original in the mid-portion. The first three frequencies for the flexural modes as predicted by the finite element model with beam elements are 24.3, 97.5 and 
$220.0 \mathrm{~Hz}$ respectively. Corresponding frequencies as predicted by the first order perturbation equation are $24.9,99.8$ and $225 \mathrm{~Hz}$.

The above set of equations, though approximate and simple and based on a first-order perturbation theory, affords insights into some concepts, summarized as follows:

(a) The defect in a beam manifested in the form of a reduction in its fundamental frequencies is a function of (a) position of damage $\left(l_{0}\right)$, (b) extent of damage $\left(2 b_{0}\right)$ and magnitude of damage, $\alpha$.

(b) The position of damage is such that it is sensitive to a certain set of frequencies only. A simple example is the invariance of even-numbered modes to a centrally situated damage.

(c) The lower-bound of the reduced frequency for a magnitude of damage $\alpha$ is at best $\sqrt{\alpha}$, when the reduction in $E I$ is full and widespread. In all other cases it is less than this value. However, for total reduction in EI, mode shape does not change from the original state.

(d) Therefore, change of natural frequency, as a parameter for damage is relatively less sensitive. Hence, in addition to the frequency, some other parameter has to be looked into, such that more information on damage could be elicited.

\section{Crack modelling}

Two models are available for modelling a defect or a crack in a linear structural member like a beam or column.

\subsection{Model 1}

In the case of a ductile material like steel, a crack may already exist and can start growing under cyclic loads. The zone over which the crack exists is in fact a length tending towards zero and hence a zone of reduced flexural rigidity cannot be conveniently adopted. However, a sudden change in flexural rigidity occurs and the model adopted in the present work is through idealizing a transversely cracked section with an equivalent rotational spring of particular spring stiffness. The rotational spring stiffness is a function of the ratio of the length of a crack to the depth of section. Linear elastic fracture mechanics principles could be used to establish the spring stiffness of a cracked section.

\subsection{Model 2}

In the second model, representing a reinforced concrete element, each section has a reduced $(E I)_{\text {eff }}$, depending upon the moment, axial and shear force carried by the member. Typically the moment curvature $(M-\phi)$ relationship of a reinforced concrete structural member, dominated by flexure can be estimated, using a fibre theory. Neutral-axis depth is sequentially progressed with varying concrete strains at extreme fibre, such that force equilibrium is achieved. The moment-curvature pair that gives rise to an such equilibrium state could then be estimated. This can also be calculated using reasonably valid approximated expressions, using a trilinear $(M-\phi)$ relationship, denoting the transition between cracking, yielding and ultimate moment stages. The effect of axial force could also be accounted in estimating such relationships. The slope of the moment curvature curve could then be used to estimate $(E I)_{\text {eff }}$ at a particular section depending on the moment carried by that section. A quasibrittle material like concrete, combined with reinforcing steel and having a large number of 
closely spaced cracks, under conditions of high bond stress between steel and concrete could be conveniently represented by an $(E I)_{\text {eff }}$ model, both for calculating deflections and also for computing the natural frequencies of a flexure-dominated beam.

\section{Mathematical foundations of wavelet transforms}

Wavelets are localized and compact signals, oscillating for a few cycles about a zero mean. A basic wavelet function can have time or space as its independent variable. For damage identification purposes, space (length) is used as the independent variable. In time-frequency analysis, time is used as the independent variable. The basic wavelet function, called as 'mother wavelet', $\psi(x)$, can be stretched or compressed by ' $a$ ' and translated in space by ' $b$ ', to generate a set of basis functions.

$$
\psi_{a, b}(x)=(1 / \sqrt{a}) \psi((x-b) / a) .
$$

Coefficients of continuous wavelet transform (CWT) of any function are obtained by integrating the dilated and translated mother wavelet with the given function for the entire space.

$$
C(a, b)=(1 / \sqrt{a}) \int_{-\infty}^{\infty} f(x) \cdot \psi((x-b) / a) \mathrm{d} x
$$

The wavelet transform results in coefficients that indicate how well a wavelet function of a particular dilation and translation correlates with the given function. Sharp and sudden variations in $f(x)$ result in large wavelet coefficients and hence the localized singularity in space or time can be captured. Unlike a Fourier transform, a one-dimensional function results in two-dimensional wavelet coefficients. In Fourier analysis, any function defined within a range of span or time can be written as the sum of sinusoidal functions of different periods (integral multiples of the given period) and whose amplitudes depend on the correlation of the original waveform with the particular sinusoid. A wavelet transform is similar to the Fourier transform, but the original function is the combination of many dilated and translated wavelets in time or space. A waveform reconstruction is possible using the mother wavelet and this is mathematically stated as,

$$
\frac{1}{K_{\psi}} \int_{-\infty}^{\infty} \int_{-\infty}^{\infty} C(a, b) \cdot \psi_{a, b}(x) \cdot \mathrm{d} b \cdot \frac{\mathrm{d} a}{a^{2}} .
$$

It can be proved that CWT is redundant and it is not necessary to use the full domain of $C(a, b)$ to reconstruct the original waveform. Instead of a continuum of dilations and translations, discrete values can be used. Dilation and translation parameters are defined as $a=2^{j}$ and $b=k 2^{j}$, where $j$ and $k$ are varying integers. Discrete wavelet transform (DWT) may be defined as,

$$
C_{j, k}=2^{-j / 2} \int_{-\infty}^{\infty} f(x) \cdot \psi\left(2^{-j} \cdot x-k\right) \mathrm{d} x .
$$

Similarly, signal reconstruction of DWT is defined by the following equation

$$
f(x)=\sum_{j=-\infty}^{\infty} \cdot \sum_{k=-\infty}^{\infty} C_{j, k} \cdot 2^{-j / 2} \cdot \psi\left(2^{-j} \cdot x-k\right) .
$$


Suppose that wavelet transform is available only for small scales, another function $\phi(x)$, called scaling function, has to be introduced. A scaling function may not exist for every wavelet, while existence of the scaling function is a pre-requisite for computation of fast wavelet transforms (FWT). It is possible to re-construct the original function as the sum of its approximations at level $J$ plus all its details up to the same level:

$$
f(x)=A_{j}(x)+\sum_{j \leq J} D_{j}(x) .
$$

Equation (6) is actually part of a larger equation, derived for multi-resolution analysis, stated as,

$$
f(x)=\sum_{j=-\infty}^{J}\left(\sum_{k=-\infty}^{\infty} c D_{j}(k) \cdot \psi_{j, k}(x)\right)+\sum_{k=-\infty}^{\infty} c A_{j}(k) \cdot \phi_{j, k}(x) .
$$

In the above equation, the first part is the detail function and the second part is the approximation function. In other words,

$$
\begin{aligned}
& D_{j}(x)=\sum_{k=-\infty}^{\infty} c D_{j}(k) \cdot \psi_{j, k}(x), \\
& A_{j}(x)=\sum_{k=-\infty}^{\infty} c A_{j}(k) \cdot \phi_{j, k}(x) .
\end{aligned}
$$

The above functions reiterate that the original function at any level $J$, can be decomposed into an approximation function, (typically of low frequency) and a number of detailed functions up to level $J$ (typically of higher frequencies). Perturbations and singularities, in the form of sudden variations, are captured in the detail (D) functions. Unperturbed original waveforms are retained through approximate (A) functions.

\section{Mode shapes of a simply supported beam with a central defect}

The simply supported beam is of $5 \mathrm{~m}$ span and has a defect at the centre (figure 2). The defect is idealized as a reduced $(E I)_{\text {eff }}$ having a $50 \%$ reduction as compared to the rest of the beam. The reduced $E I$ is spread over a length of $0.25 \mathrm{~m}$ (5\% of the total length). The beam is idealized as a two-dimensional beam with 200 elements for the span length. The length of a sub-element is thus $0.025 \mathrm{~m}$. A sub-space iteration technique is used to extract the first 15 modes of the beam with a lumped mass formulation. The rotation and curvature

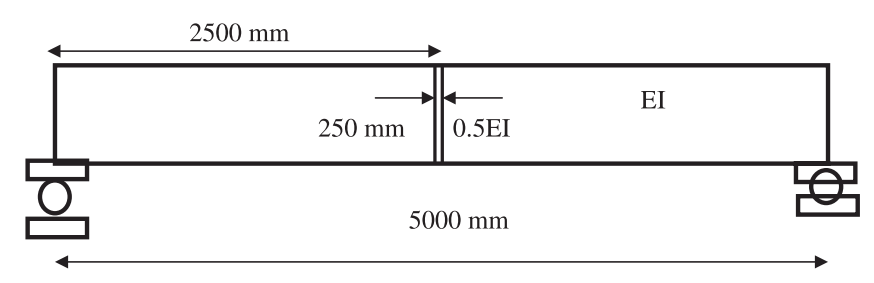

Figure 2. Example of a simply supported beam used in the study. 


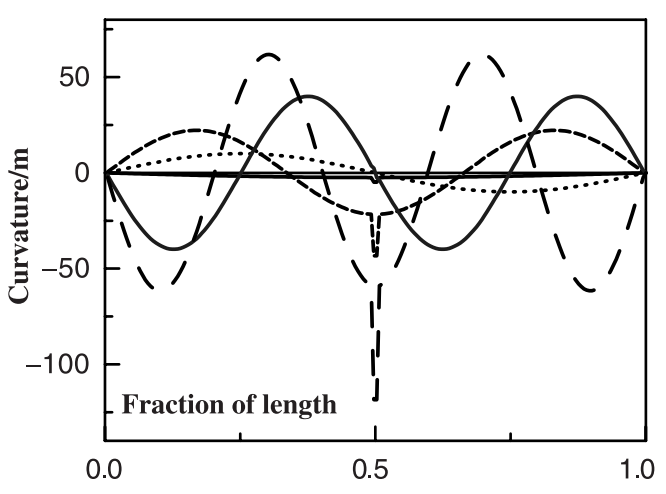

Figure 3. Curvature variation of defective beam for fundamental and higher modes.

variation of the mode shapes are also obtained. Figure 3 shows the curvature mode shapes for the fundamental as well as higher modes. Since the reduction in $E I$ is half, curvature shoots up by a factor of 2 . Higher modes exhibits higher curvatures as the second derivative of the mode shape varies at a faster rate compared to the displacement. Curvature is computed using the following difference equation,

$$
\frac{M}{E I}=-\frac{\mathrm{d}^{2} y}{\mathrm{~d} x^{2}}=\frac{y_{n+1}-2 y_{n}+y_{n-1}}{\Delta h^{2}}=\frac{\theta_{n+1}-\theta_{n}}{\Delta h}
$$

The idea of increased curvature for higher modes is understood by the following logic, using the example of the mode shape of a simply supported beam:

$$
\begin{aligned}
\phi_{n} & =A \sin (\pi n x / l), \\
\phi_{n}{ }^{\prime \prime} & =-A(\pi n / l)^{2} \sin (\pi n x / l), \\
\phi_{n}{ }^{\prime \prime} & =-A(\pi /(l / n))^{2} \sin (\pi n x / l) .
\end{aligned}
$$

For example, the fifth mode of a beam will have a curvature equal to 25 times the first mode. The other way of visualizing this is that if the defect is spread over a length of $\gamma . l$ for the original beam, the same beam will have a pseudo span reduced $N$ times, when vibrating in the ' $N$ th' mode. In other words, there is an apparent increase of $N$ times in the ratio of the defective length to the pseudo-span length when measurements are carried out at the $N$ th mode. Further, curvature jumps up as a square of this ratio. Wavelet multi-resolution analyses of the displacement and rotation mode shapes are also performed and the results are shown in Figures 4 to 9. Daubechies (DB4) and bi-orthogonal (BIOR 6.8) family of wavelets, available in MATLAB wavelet tool-box suite are used for the computation of the approximate and detail functions. Two level multi-resolution analyses is found to be sufficient to capture the disturbances in the deformation profile

The ratio of detail D2 magnitudes as compared to the original signal values for displacement mode shapes are $0.02,0.1$ and $0.4 \%$ respectively for the first, third and fifth modes. Similarly, the ratio of detail D2 magnitudes as compared to the original signal values for rotation mode shapes are $0.5,1.0$ and $2.0 \%$ respectively for the first, third and fifth modes. These perturbations in the detail coefficients are similar to the high frequency content of a time frequency signal and, in actual measurements, a minimum threshold signal is always required such that it is above the electrical noise of the measuring instrument. In this context, the displacement perturbations, particularly for lower modes may not cross the noise threshold 


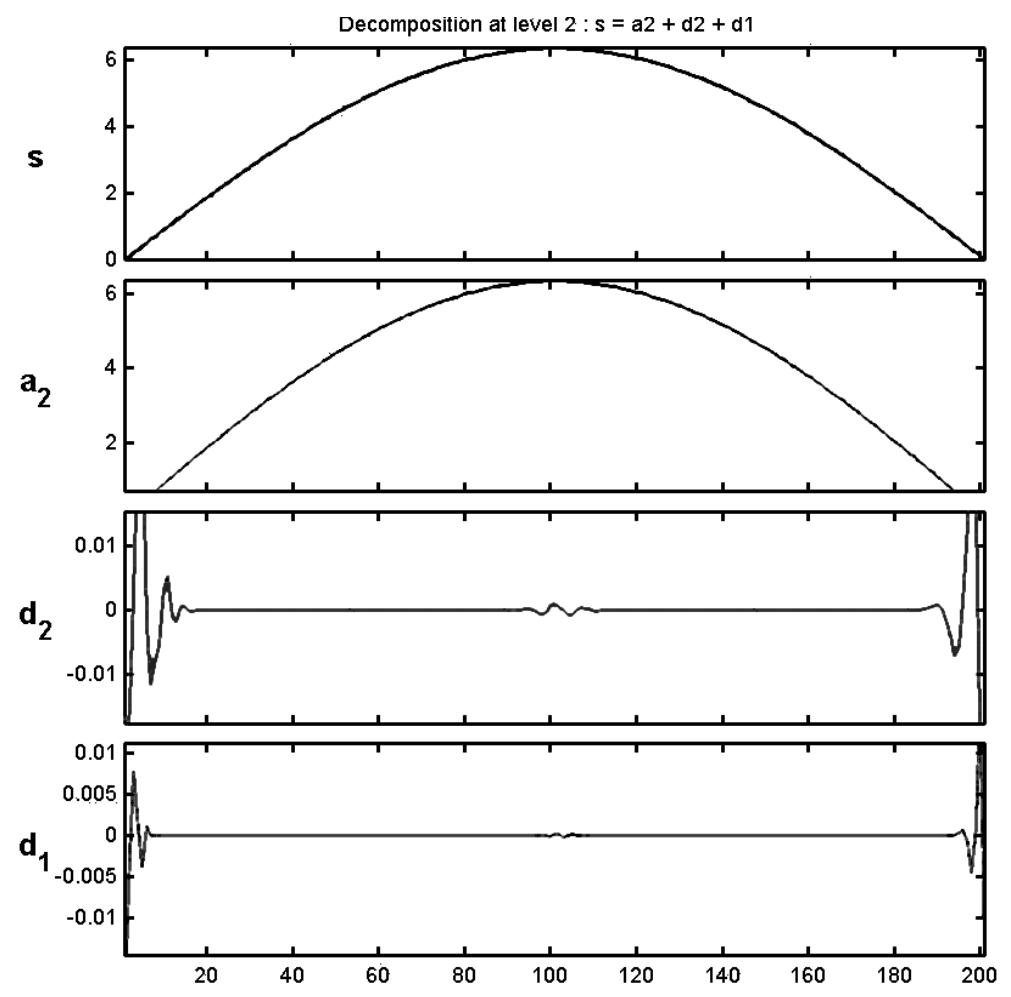

Figure 4. Wavelet multi-resolution analysis of the displacement mode of the defective beam for mode-I.

values of the measuring instrumentation. Even modes do not show any perturbations either in displacement or rotation mode shapes as the defect is situated at the displacement node position of these modes.

\section{Static response of the beam with an off-centric defect}

Static-deflected shape of the same beam is computed for a concentrated load applied at the centre. A concentrated load gives rise to a linear bending moment diagram and there is a third-derivative discontinuity (in shear force variation) in the displacement profile. Hence the defect is shifted to a position of 0.21 from the support. Here too, the defect is idealized as a reduced $(E I)_{\text {eff }}$ having a $50 \%$ reduction as compared to the rest of the beam. The reduced $E I$ is spread over a length of $0.25 \mathrm{~m}$ ( $5 \%$ of the total length), with its centre located at 0.21 from the support. The beam is idealized as a two-dimensional beam with 200 elements for the span length and the length of a sub-element is kept at $0.025 \mathrm{~m}$. The curvature variation is plotted in figure 10 and it exactly follows the bending moment variation of the simply supported beam. The defect shows up as doubled up curvature value at the 0.21 position. Wavelet analysis is performed for both the displacement as well as rotation mode shapes (figure 11). The detail coefficients hardly show up in the displacement profile whereas the rotation profile shows up small disturbances. A disturbance occurs below the concentrated load also due to the thirdderivative discontinuity of the displacement. This particular problem is unlikely to occur 


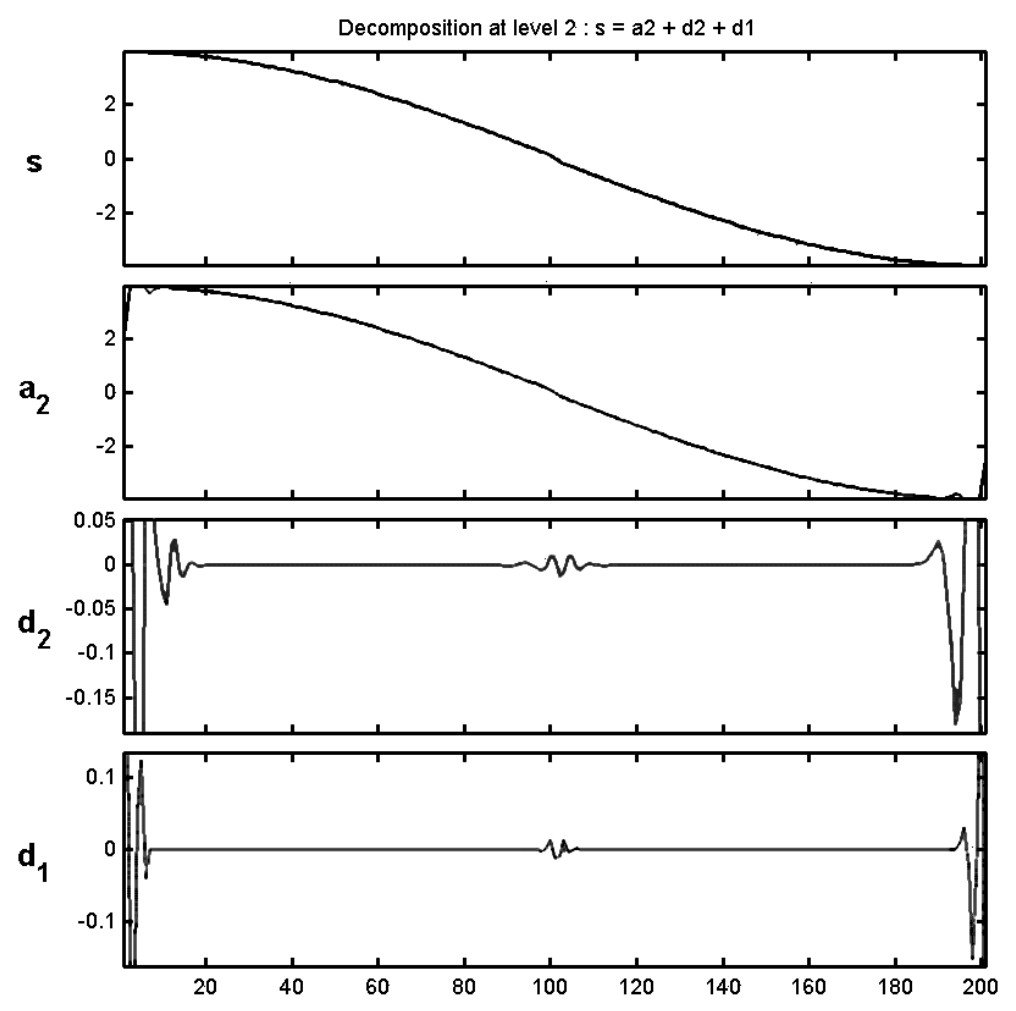

Figure 5. Wavelet multi-resolution analysis of the rotation mode of defective beam for mode-I.

for dynamic loads as the loading is due to distributed effect of mass. The arguments of the previous case hold true in this case also and the displacement perturbations may sometimes be buried within the random noise field of the measuring instrumentation.

\section{Dynamic response of a steel beam with a central crack (model 1)}

A model which uses a reduced $E I$ for modelling a local damage is sometimes good enough, but a real cracked section, with the length of the crack tending towards zero, is analogous to Dirac's delta function used in representing a singularity, with the integrated damage value being finite. As length of the crack is small, the curvature jump is infinite, but rotation shows a definite discontinuity across the crack. The transversely located physical crack existing in a steel structure for part of the depth and potentially growing in mode-I fashion due to cyclic effects can be modelled as a rotational spring. It is possible to determine the rotational spring stiffness of a cracked section, if the ratio of the depth of the crack with reference to the depth of the section is known, using principles of liner elastic fracture mechanics.

Figure 12 shows the modelling of such a crack using a rotational spring element. If the spring is very stiff, rotation difference across the plane of the crack is preserved and vice versa, there exists a discontinuity of slopes. The boundary conditions for these crack modelling include:

(a) Moment, shear force and displacement continuity across the crack section 


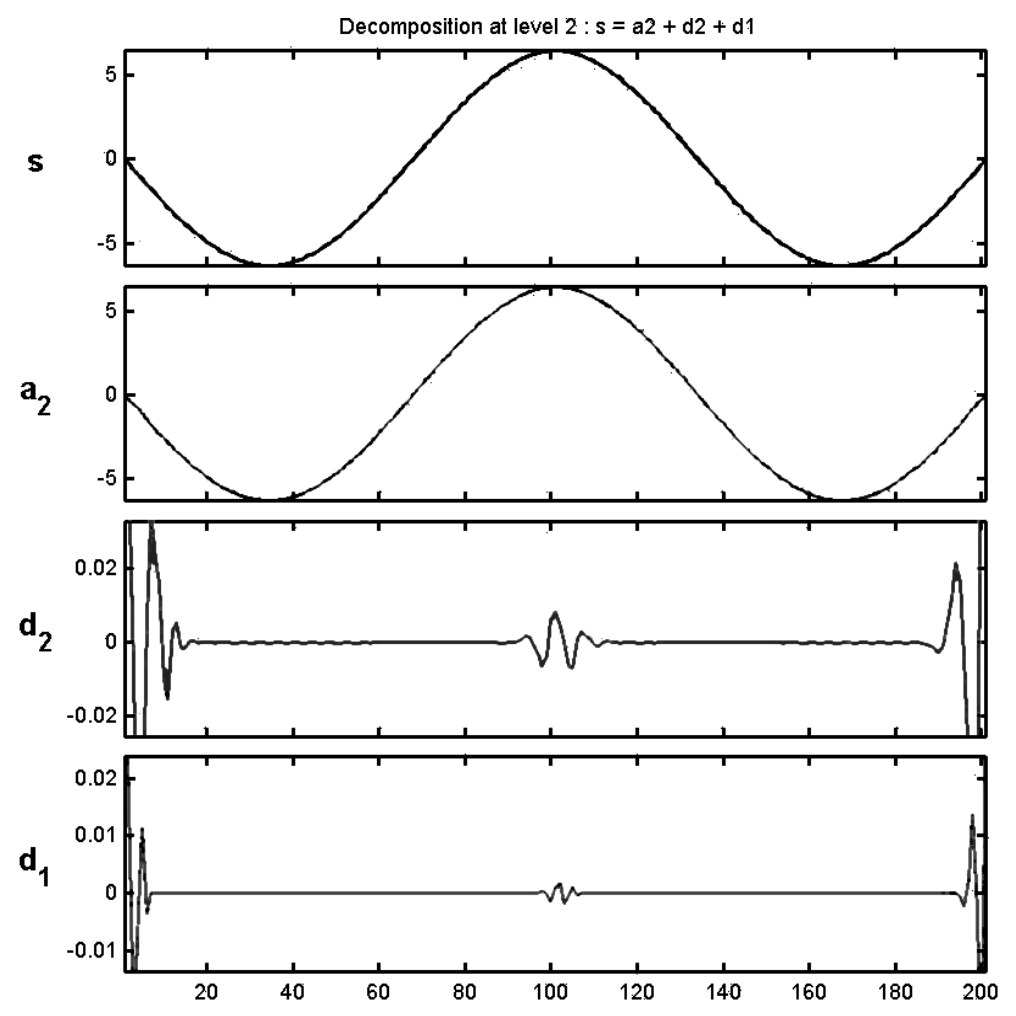

Figure 6. Wavelet multi-resolution analysis of the displacement mode of defective beam for mode-III.

(b) The product of the rotational spring stiffness and the difference in the rotations on the two sides of the crack is equal to the external bending moment

The computer program is modified to account for rotation springs and the spring stiffness corresponds to a partial value of depth of the crack.

Wavelet analysis results of the rotation mode shapes are shown in Figures 13 to 15, for the first, third and fifth modes. Detail coefficient (D1) and the related function values are more, when compared to (D2), unlike the previous cases, due to the fact that the crack is sharp and represents high frequency variation, whereas in the previous values the crack existed for larger lengths (5\%). The ratio of detail function magnitudes as compared to the original signal values for rotation mode shapes are $1.25,5.0$ and $10.0 \%$ respectively for the for the first, third and fifth modes.

It is necessary to examine the reason for the enhanced perturbation values exhibited at higher modes. Previously it is argued that the longitudinal dimension of the crack is considerable when compared to the pseudo span length of the beam at higher modes, where pseudo span length is defined as part of the length of the beam, between two consecutive curvature nodes. The results show the same trend in this model too, even though the length of the crack is nearly zero. The spring stiffness of the rotation spring, representing a transversely cracked plane is high compared to the original length of the beam. In higher modes, the length of beam between the crack and the adjacent point of zero curvature falls $N$ times and thus the ratio of 


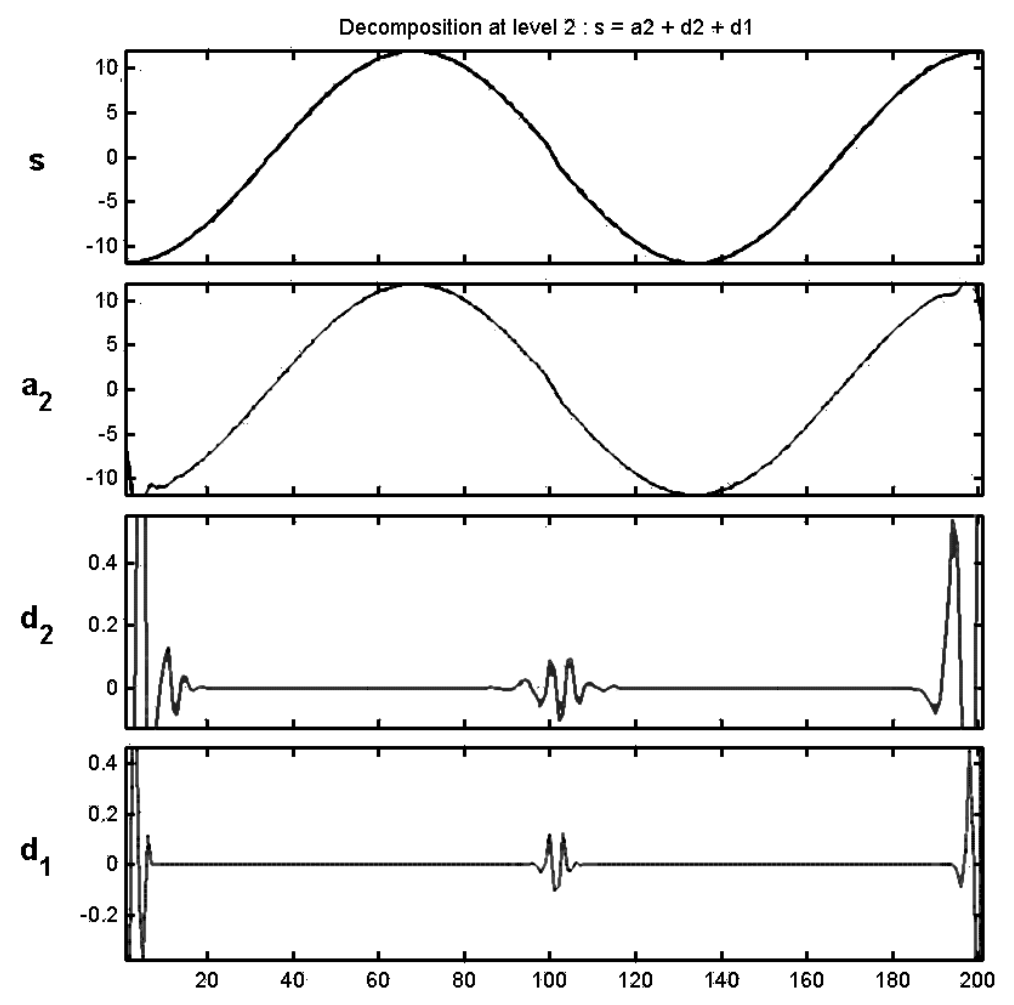

Figure 7. Wavelet multi-resolution analysis of the rotation mode of defective beam for mode-III.

the crack-spring stiffness with reference to the rotation spring stiffness of the pseudo-beam also falls, thus showing more perturbation coefficients at higher modes.

\section{Damage localization and forcing curvature nodes to desired locations}

It is seen that the values of detail (D) coefficients, in a wavelet multi-resolution analysis which represent the spatial local disturbances (analogous to higher frequency variations in a time signal) are enhanced with rotation and curvature measurements and become stronger under higher modes. In the case of a simply supported beam representing a bridge beam, curvature and displacement nodes appear at pre-determined locations for mode shapes. If damage occurs at a node location (zero amplitude location) corresponding to a particular mode, then measurements have to be repeated at some other mode so that the region of interest falls in the anti-node location. Towards solving this problem, a method is devised such that the point of interest lies in the peak region of curvature variation. The method is illustrated using two examples for a simply supported beam, but could be easily extended to any other beam with arbitrary boundary conditions. However, the sacrifice to be made for picking up the peak curvature position at any arbitrary location is that the vibration shape corresponds to excitation frequency, which is off-resonance and in-between two natural frequencies. This requires the excitation force to be higher, to get the same high amplitudes at resonance.

Steady state deflected shape of a beam vibrating between $N$ and $(N+1)$ th mode of a beam is given using the following expression, assuming that modes other than $N$ and $N+1$ have 


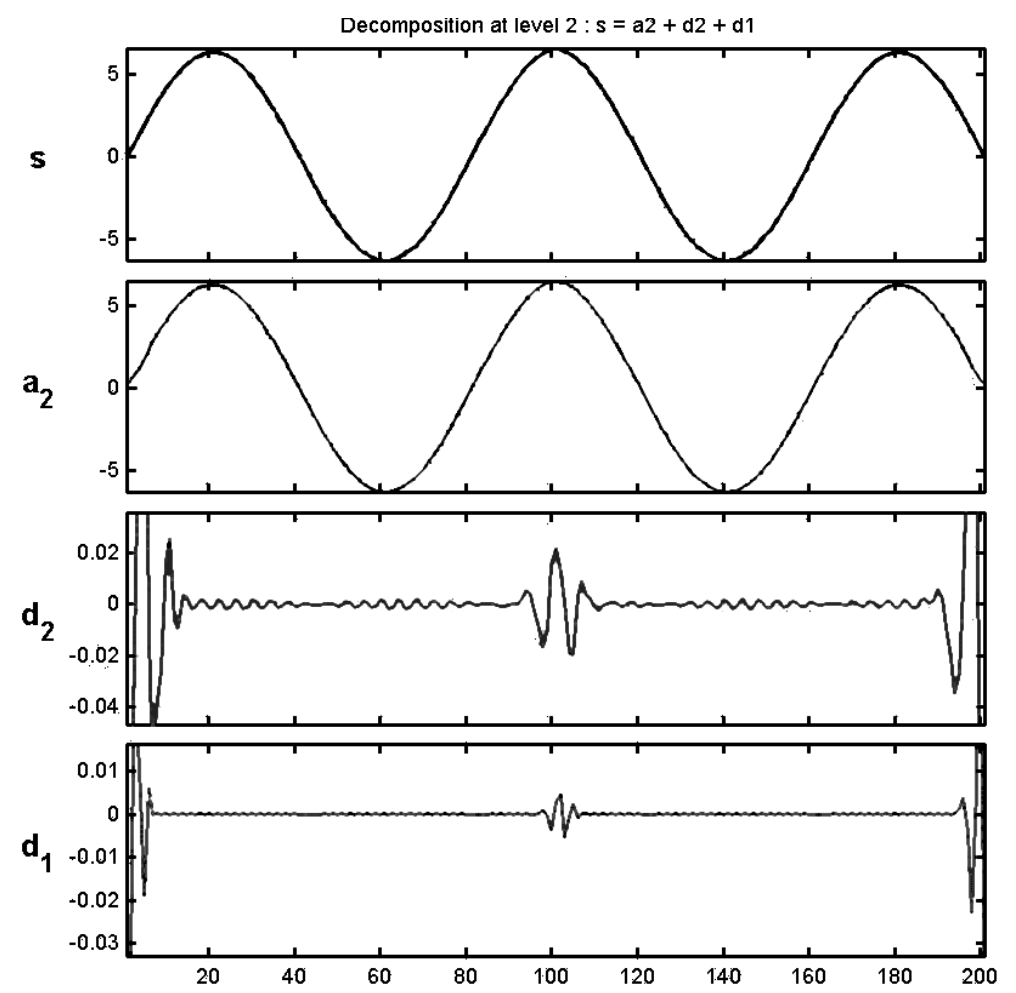

Figure 8. Wavelet multi-resolution analysis of the displacement mode of defective beam for mode-V.

reduced magnification factors and do not participate in the response. Away from resonances, the effect of damping is negligible and this effect is also not taken into account. The excitation is applied at a distance of $c$ from the left end and the response is measured at distances $a$ and $b$ from the left end. $\omega_{1}$ is the first flexural frequency of the system and $\omega$ is the excitation frequency.

$$
\begin{aligned}
y(x)= & \frac{1}{0 \cdot 5 M}\left[\frac{1}{\left(N^{4} \omega_{1}^{2}-\omega^{2}\right)} \sin \left(\frac{N \pi c}{l}\right) \sin \left(\frac{N \pi x}{l}\right)\right. \\
& \left.+\frac{1}{\left((N+1)^{4} \omega_{1}^{2}-\omega^{2}\right)} \sin \left(\frac{(N+1) \pi c}{l}\right) \sin \left(\frac{(N+1) \pi x}{l}\right)\right], \\
y^{\prime \prime}(x)= & \frac{\pi^{2}}{0 \cdot 5 M l^{2}}\left[\frac{N^{2}}{\left(N^{4} \omega_{1}^{2}-\omega^{2}\right)} \sin \left(\frac{N \pi c}{l}\right) \sin \left(\frac{N \pi x}{l}\right)\right. \\
& \left.+\frac{(N+1)^{2}}{\left((N+1)^{4} \omega_{1}^{2}-\omega^{2}\right)} \sin \left(\frac{(N+1) \pi c}{l}\right) \sin \left(\frac{(N+1) \pi x}{l}\right)\right] .
\end{aligned}
$$

Assuming that curvature nodes lie at positions at $a$ and $b$ the from origin, gives rise to the condition that $y^{\prime \prime}(a)=y^{\prime \prime}(b)=0$. 

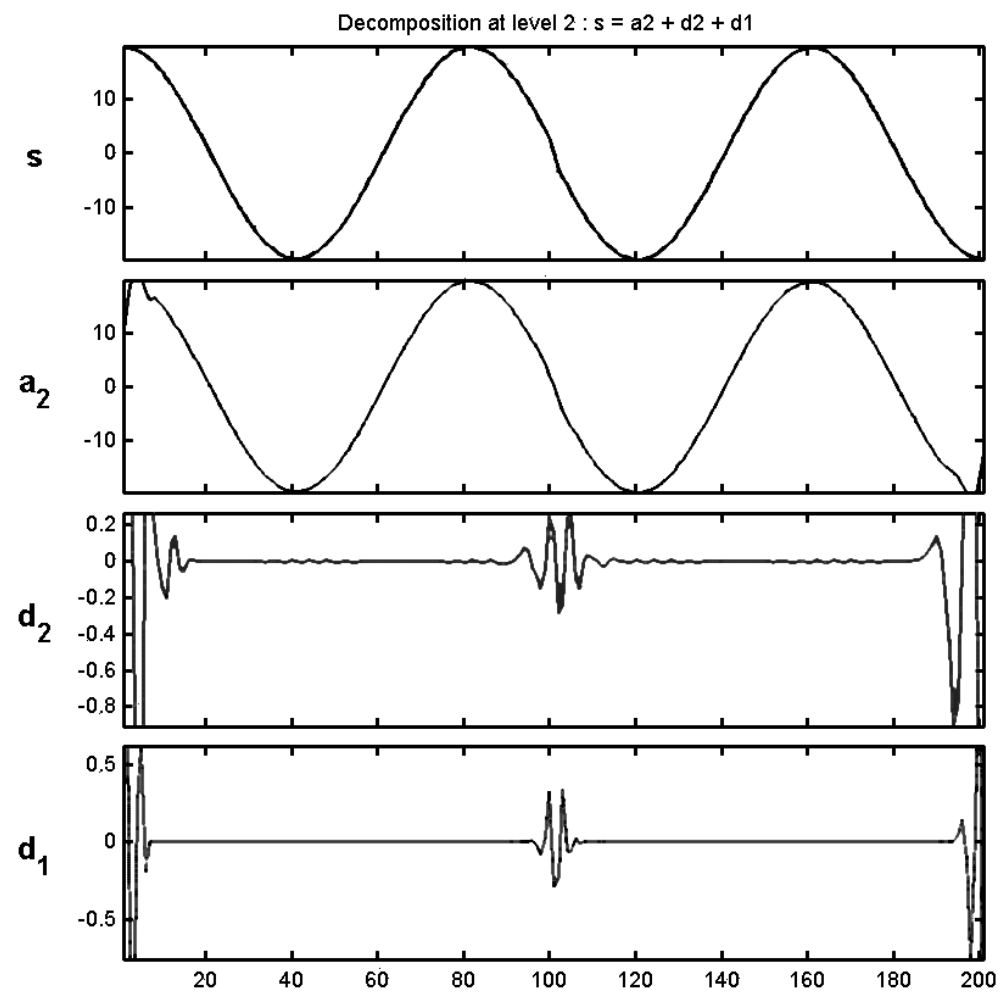

Figure 9. Wavelet multi-resolution analysis of the rotation mode of defective beam for mode-V.

Simplifying (25)

$$
1+\frac{\sin ((N+1) \pi c / l) \sin ((N+1) \pi a / l)\left(N^{4} \omega_{1}^{2}-\omega^{2}\right)(N+1)^{2}}{\sin (N \pi c / l) \sin (N \pi a / l)\left((N+1)^{4} \omega_{1}^{2}-\omega^{2}\right) N^{2}}=0 .
$$

Similarly for the second condition,

$$
1+\frac{\sin ((N+1) \pi c / l) \sin ((N+1) \pi b / l)\left(N^{4} \omega_{1}^{2}-\omega^{2}\right)(N+1)^{2}}{\sin (N \pi c / l) \sin (N \pi b / l)\left((N+1)^{4} \omega_{1}^{2}-\omega^{2}\right) N^{2}}=0
$$

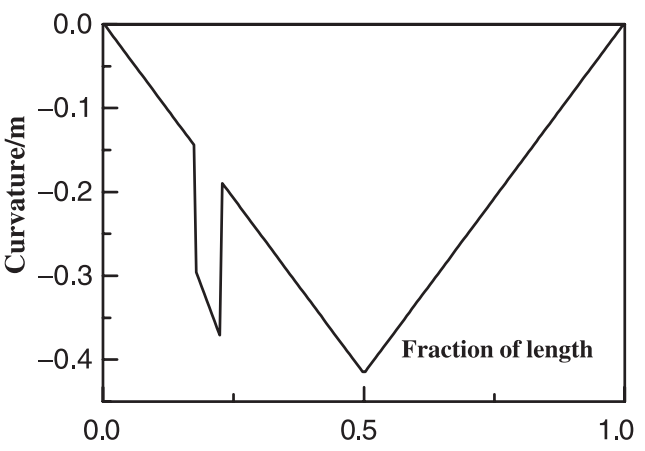

Figure 10. Curvature variation for the static load case. 


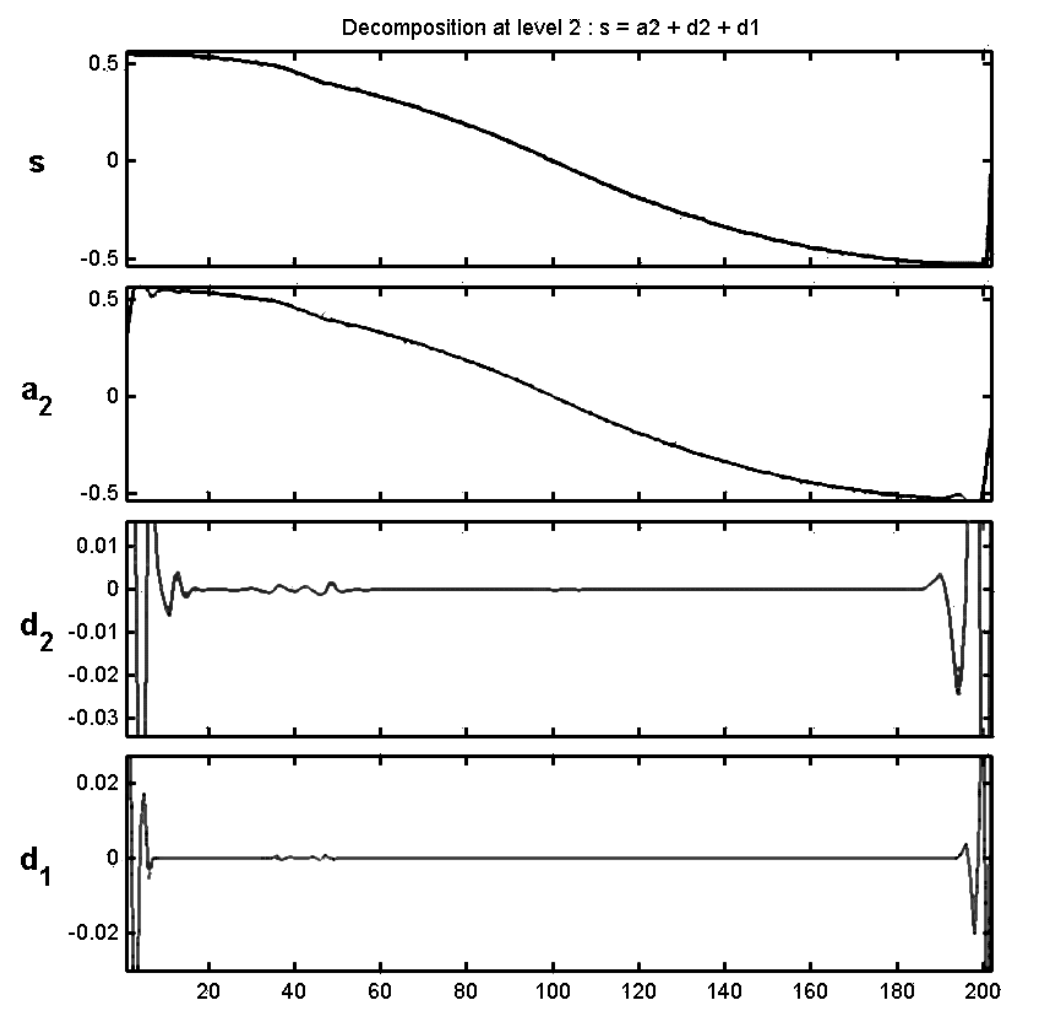

Figure 11. Wavelet multi-resolution analysis of the rotation of static deflected shape.

Assuming further simplification like

$$
\begin{aligned}
& \sin \left(\frac{(N+1) \pi a}{l}\right) / \sin \left(\frac{N \pi a}{l}\right)=\sin \left(\frac{(N+1) \pi b}{l}\right) / \sin \left(\frac{N \pi b}{l}\right)=\beta, \\
& \frac{\sin (N \pi c / l)\left((N+1)^{4} \omega_{1}^{2}-\omega^{2}\right) N^{2}}{\sin ((N+1) \pi c / l)\left(N^{4} \omega_{1}^{2}-\omega^{2}\right)(N+1)^{2}}=-\beta .
\end{aligned}
$$

The above sets of equations suggest that once the position of $a$ is determined, $b$ is also automatically known, satisfying (28). Further, if the excitation point $c$ is known, (29) could be used to determine the excitation frequency $(\omega)$.

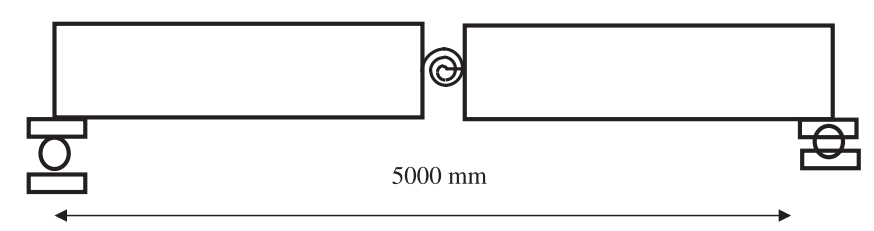

Figure 12. Model to represent a transverse crack in a steel beam. 


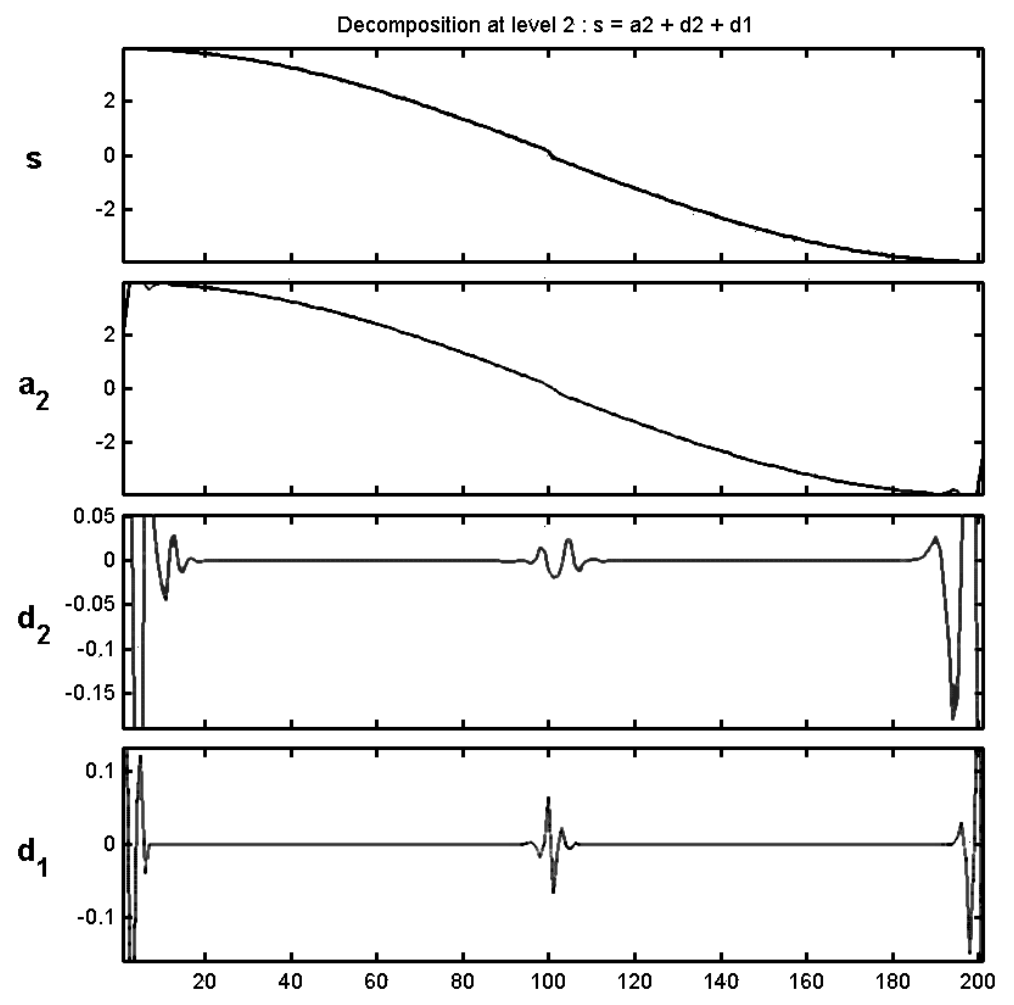

Figure 13. Wavelet multi-resolution analysis of the rotation mode shape for steel beam - mode-I.

For evaluating the localized span, at the centre of which the curvature is maximized, (28) is written as,

$$
\begin{aligned}
\sin ((N+1) \pi a / l) & =-\sin ((N+1) \pi b / l), \\
{[(N+1) \pi a / l]+\pi } & =[(N+1) \pi b / l], \\
\sin (N \pi a / l) & =-\sin (N \pi b / l), \\
(N \pi a / l)+\pi & =(N \pi b / l) .
\end{aligned}
$$

Solving (28) in this manner is not exact, nevertheless, it throws light on the approximate value of the positions of $a$ and $b$ for particular pair of modes $N$ and $N+1$.

Two different equations yield two different values relating $a$ and $b$. They are,

$$
\begin{aligned}
{[(N+1) \pi a / l]+\pi } & =(N+1) \pi b / l, \\
(b-a) & =l /(N+1), \\
(N \pi a / l)+\pi & =N \pi b / l, \\
(b-a) & =l / N, \\
(b-a)_{\text {ave }} & =\frac{1}{2}[(l /(N+1))+(l / N)] .
\end{aligned}
$$




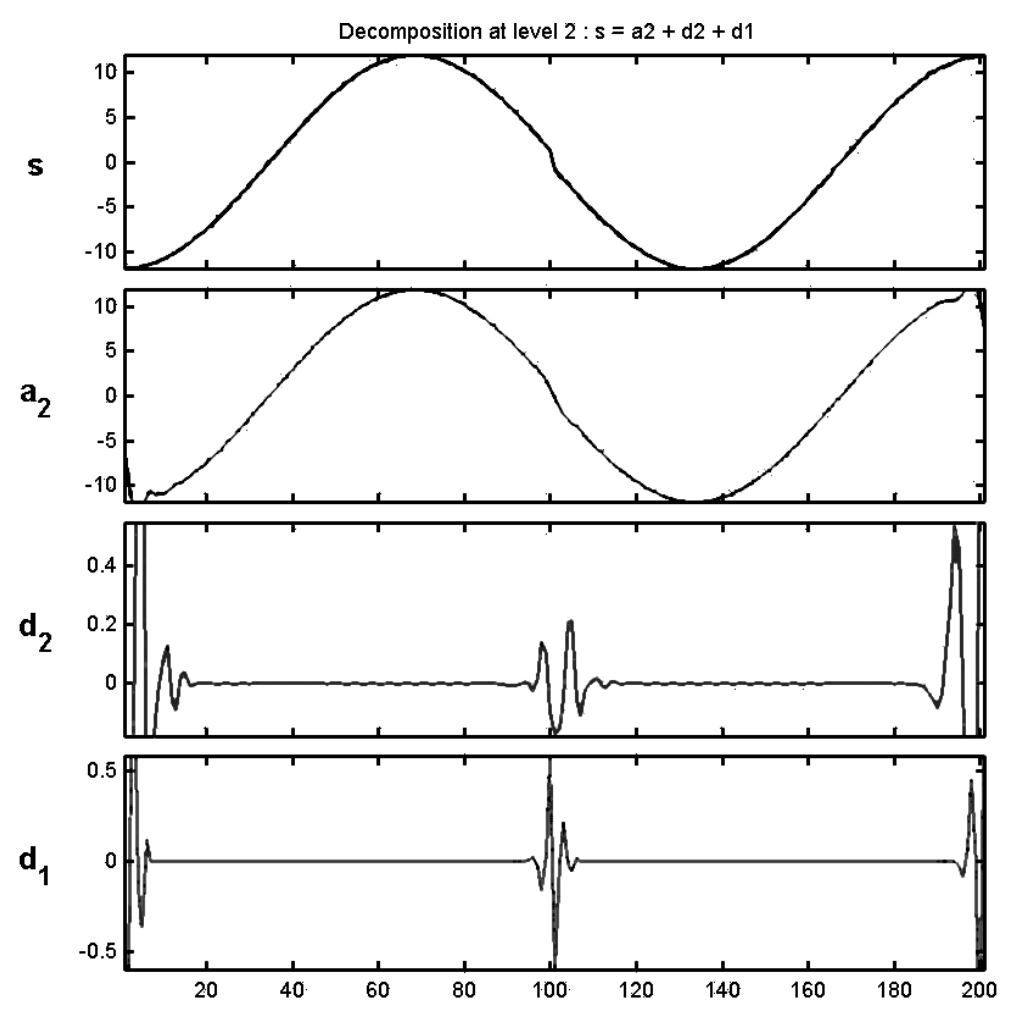

Figure 14. Wavelet multi-resolution analysis of the rotation mode shape for steel beam - mode-III.

Hence the value of pseudo span is approximated as the average of the pseudo spans corresponding $(N+1)$ and $N$ th modes.

After finding the value of $a$ and $b$, the excitation location $c$ is varied, such that $y^{\prime \prime}((a+b / 2))$ is maximized.

\subsection{Case study -1}

It is required to create an arbitrary span, with damage location at $(a+b) / 2=0.4$ for $N=2$. The defect is introduced in the form of $50 \%$ reduction in $(E I)$ for a length of $0.25 \ell$ and situated at 0.41 . Average span is given by (31), and predicted as 0.417 . Hence $a$ and $b$ values are predicted as 0.1921 and 0.6081 . $\beta$ has to be found for these values of $a$ and $b . \beta$ is found as 1.03 for $a=0.192$ and 0.86 for $b=0.608$. After slight adjustments, $a$ and $b$ are found to be $0 \cdot 2$ and $0 \cdot 6$, which yield the same value of $\beta(=1 \cdot 0)$.

The curve $\sin [(N+1) \pi a / l] / \sin (N \pi a / l)$ is plotted and shown in figure 16 . The values of curvature for different locations of $c$ (excitation position) are then obtained and plotted in figure 17. Excitation frequencies corresponding to different excitation locations are plotted in figure 18. As the position of excitation force and the frequency is known, an actual steady state dynamic analysis is performed with ten flexural modes participating in the response. The curvature variations for both normal and defective beams are then plotted in figure 19 . Figure 20 shows the wavelet multi-resolution analysis of the steady state rotation profile of the investigated beam in case study -1 . 


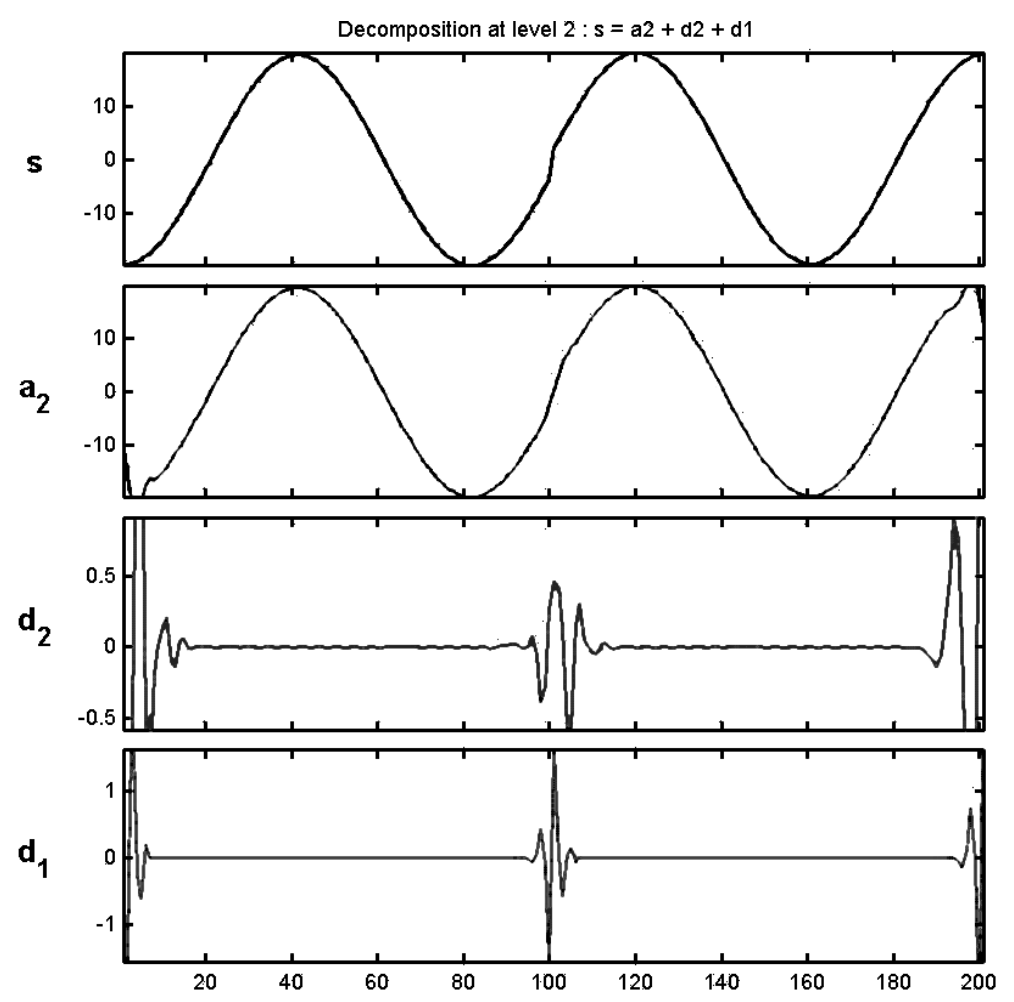

Figure 15. Wavelet multi-resolution analysis of the rotation mode shape for steel beam - mode-V.

\subsection{Case study -2}

This is similar to case study -1 , except that damage location is at $0 \cdot 21$ and the pseudo span length, which has the damage location at its centre has to be at $0 \cdot 21 . N$ is chosen as 5 and the damage location is close to the node position of both fifth and sixth modes. Average span is calculated as 0.181 . After a few trials and from the curve showing the $\beta$ variation (figure 21), $a$ and $b$ values are fixed as 0.1 and 0.2755 respectively giving rise to a constant value of $\beta(0.9511)$. The centre of this curve is $0 \cdot 191$, which is near the assumed damage position. Curvature variation using the $N$ and $(N+1)$ th modes is plotted in figure 22 . The corresponding excitation frequencies are shown in figure 23. Using these values of excitation location and frequency, actual dynamic analysis, using ten flexural modes is performed and the resulting curvature variations along the span are shown in figure 24 , for both the normal and defective beams. The defect is introduced in the form of $50 \%$ reduction in $(E I)$ for a length of $0.25 \mathrm{~m}$ and situated at $0 \cdot 21$. Figure 25 shows the wavelet multi-resolution analysis of the steady state rotation profile of the investigated beam, in case study -2 . The validity of the developed method is thus established.

\section{Conclusions}

An equation is developed for computing the natural frequencies of a damaged beam using first-order perturbation theory. The equation is made use of in proving that natural frequency 

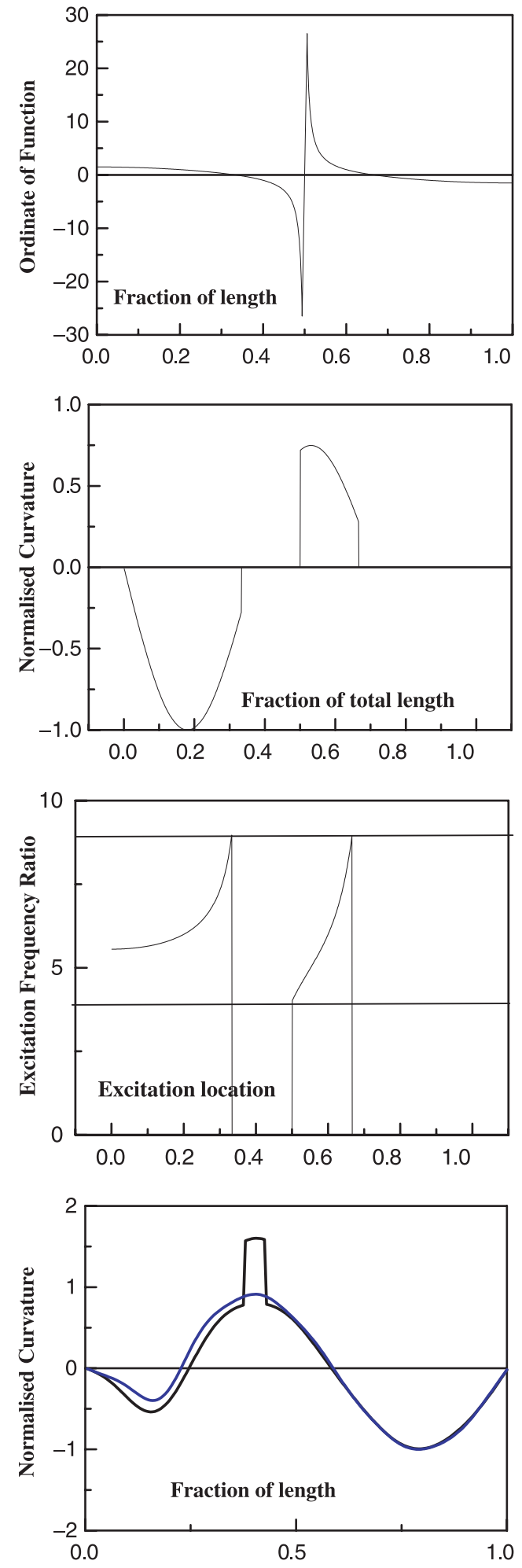

Figure 16. Variation of $\beta$ for $N=2$ (case study - 1).

Figure 17. Curvature distribution for different excitation positions (case study - 1).

Figure 18. Variation of excitation frequencies for different excitation locations (case study - 1).

Figure 19. Variation of curvatures for defective and normal beams (case study - 1). 


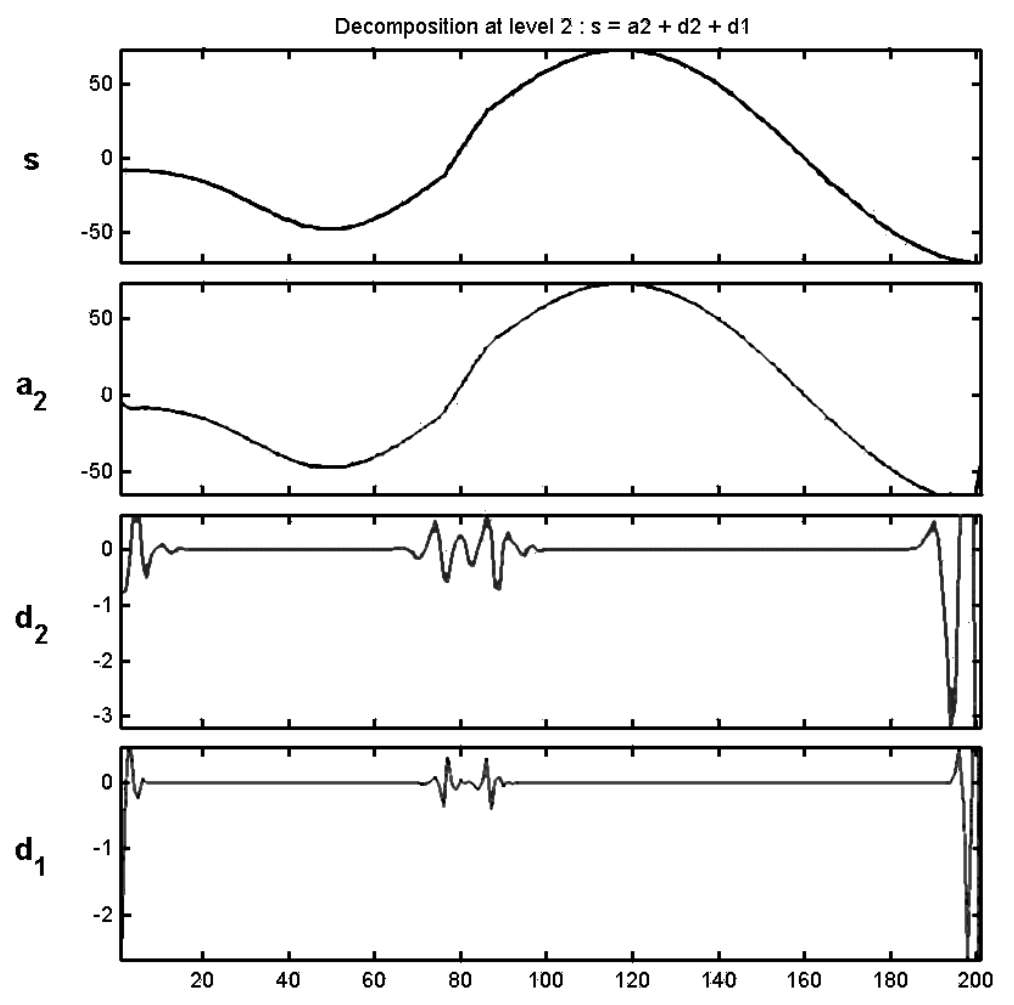

Figure 20. Wavelet multi-resolution analysis of rotation profile (case study -1).

is a relatively insensitive parameter to assess damage and hence there is a need to choosing additional parameters or schemes to establish damage in a structural element. Free vibration mode shape and static deflected shape of a damaged structural beam element are computed and subjected to multi-resolution wavelet analysis. The damage modelling is done by using a rotational spring to model the crack location. This model is typically used for idealizing cracked locations in steel beams and the appropriate spring stiffnesses are derived using fracture mechanics principles.

Daubechies (DB4) and bi-orthogonal (BIOR 6.8) family of scaling and wavelet functions are used for the computation of the approximate (A) and detail (D) functions. Two-level multi-resolution analysis is found to be sufficient to capture the disturbances in the deformation profile. Displacement, rotation and curvatures are used as damage indicators and their relative performance suggests that rotation and curvatures are better damage indicators as compared to displacements. Rotation mode shapes are not that difficult to measure and laser-based non-contact vibration meters employing Doppler effects are already in vogue, which can scan a small area and report the local dynamic rotations. Higher order modes, are particularly suitable and those modes where the curvature shows peaks are ideal ones for damage detection. It may be surmised that static deflected shapes and lower mode displacement profiles may show disturbances in the wavelet detail coefficients but may possibly get submerged in the instrument noise of the measuring system. 

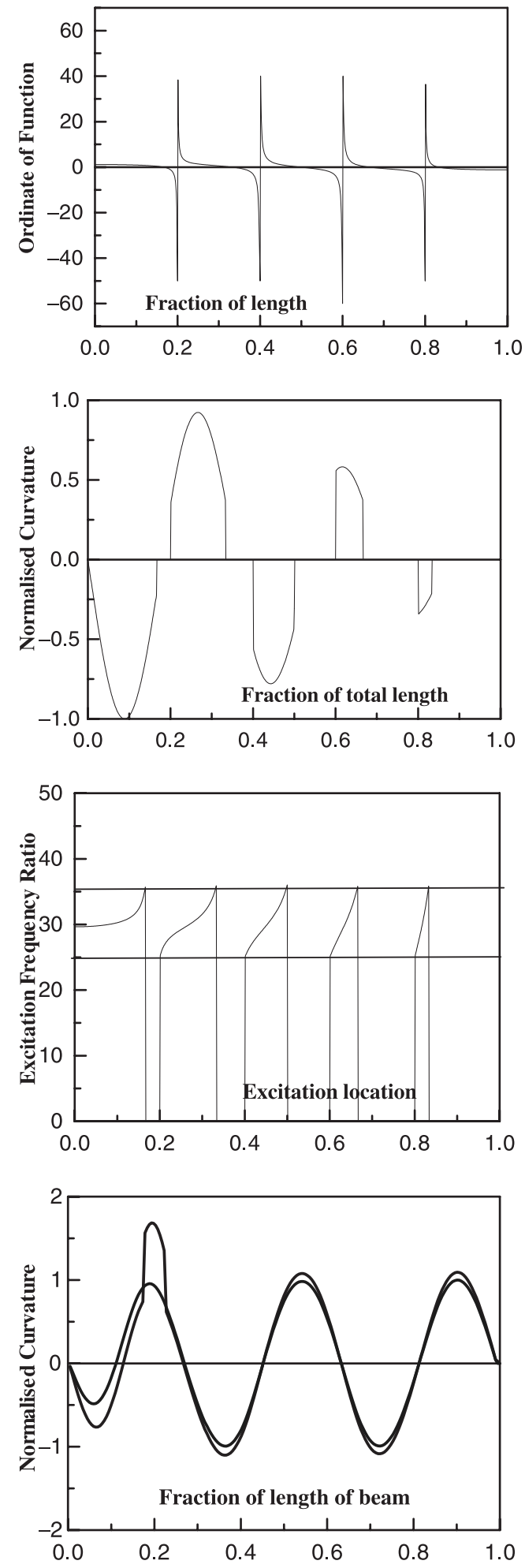

Figure 21. Variation of $\beta$ for $N=5$ (case study - 2).

Figure 22. Curvature distribution for different excitation positions (case study - 2).

Figure 23. Variation of excitation frequencies for different excitation locations (case study -2 ).

Figure 24. Variation of curvatures for the defective and normal beams (case study - 2). 

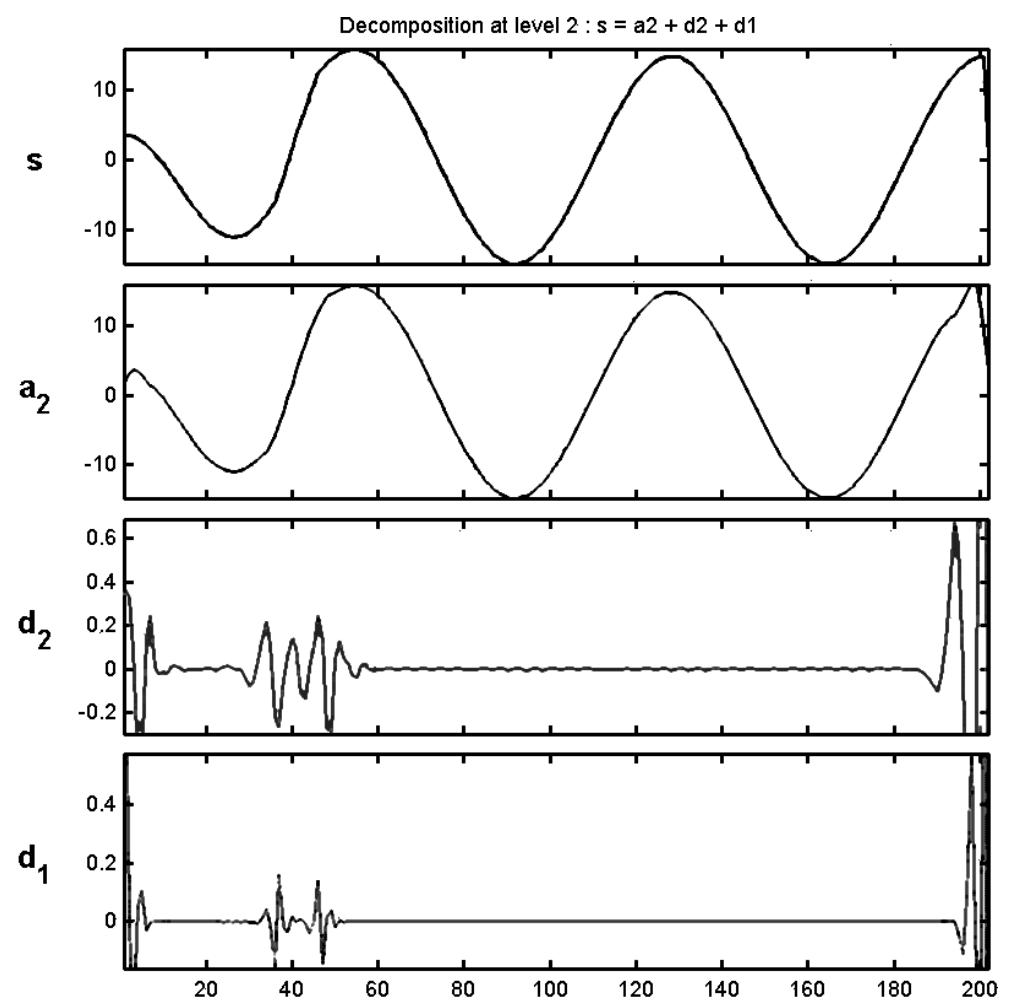

Figure 25. Wavelet multi-resolution analysis of rotation profile (case study - 2).

A method is devised such that curvature nodes could be forced at arbitrary points of interests and the mid-locations of these pseudo spans can be observed for potential cracks. A simplified set of equations is developed assuming contribution only from the two adjacent modes. This method could be adopted if a curvature peak is automatically not occurring in an anti-node location of a resonating beam. The method is illustrated for a simply supported beam and could be extended to any arbitrary linear structural member provided mode shape and frequency information are available for higher modes.

\section{List of symbols}

$a, b$

$A(x)$ and $D(x)$

$c$

$C(a, b)$

EI

$f_{c k}$

$K_{0}, K_{1}$

$M_{0}, M_{1}$ start and end position of pseudo span, reckoned from left end of the beam;

approximate and detail functions;

load application point from left end of the beam;

wavelet coefficients corresponding to translation $(a)$ and dilation

(b) values;

flexural rigidity of the beam section;

characteristic strength of concrete;

original and perturbed stiffness matrices;

original and perturbed mass matrices; 
$N, N+1 \quad$ integers corresponding to $N$ and $N+1$ modes;

$p_{i}^{(0)} \quad$ eigenvector of the $i$ th mode before perturbation;

$\alpha \quad$ ratio of change in flexural rigidity;

$\beta \quad$ function defined as $\sin ((N+1) \pi a / l) / \sin (N \pi a / l)$;

$\gamma \quad$ a temporary variable;

$\lambda_{i}^{(0)} \quad$ eigenvalue of the $i$ th mode before perturbation;

$\phi \quad$ wavelet scaling function;

$\psi \quad$ wavelet mother function;

$\omega \quad$ the excitation frequency;

$\omega_{1} \quad$ first flexural frequency of the system.

\section{References}

Abdel Wahab M M, Roeck G D 1999 Damage detection in bridges using modal curvatures: Application to a real damage scenario. J. Sound Vibr. 226: 217-235

Abdo M A B, Hori H 2002 A numerical study of structural damage detection using changes in the rotation of mode shapes. J. Sound Vibr. 251:

Daubechies I 1992 Ten lectures on wavelets (Philadelphia, PA: SIAM)

Gurley K, Kareem A 1999 Applications of wavelet transforms in earthquake wind and ocean engineering. Eng. Struct. 21: 149-167

Hassiotis S 2000 Identification of damage using natural frequencies and Markov parameters. Comput. Struct. 74: 365-373

Hassiotis S, Jeong G D 1993 Assessment of structural damage from natural frequency measurements. Comput. Struct. 49: 679-691

Hera A, Hou Z 2004 Application of wavelet approach for ASCE structural health monitoring benchmark studies. J. Eng. Mech. 96-104

Hou Z, Noori M, Amand R S 2000 Wavelet based approach for structural damage detection. J. Eng. Mech. 677-683

Hubbard B B 2003 The world according to wavelets 2nd edn (Hyderabad: Universities Press)

Kim H, Melhem H 2004 Damage detection of structures by wavelet analysis. Eng. Struct. 26: 347-362

Lakshmanan N, Srinivasulu P, Parameswaran V S 1991 Post cracking stiffness and damping in reinforced concrete beam elements. J. Struct. Eng. India 17: 1405-1411

Liew K M, Wang Q 1998 Application of wavelet theory for crack identification of structures. J. Eng. Mech. 152-157

Majumder L, Manohar C S 2003 A time domain approach for damage detection in beam structures using vibration data with a moving oscillator as an exciting source. J. Sound Vibr. 268: 699-716

Majumder L, Manohar C S 2004 Nonlinear reduced models for beam damage detection using data on moving oscillator - beam interaction. Comput. Struct. 82: 301-314

Mallat S A 1998 Wavelet tour of signal processing (Boston: Academic Press)

Melhem H, Kim H 2003 Damage detection in concrete by Fourier and wavelet analysis. J. Eng. Mech. 571-577

Ovanesova A V, Suarez L E 2004 Application of wavelet transforms to damage detection in frame structures. Eng. Struct. 26: 39-49

Owolabi G M, Swamidas A S J, Seshadri R 2003 Crack detection in beams using changes in frequencies and amplitudes of frequency response functions. J. Sound Vibr. 265: 1-22

Pandey A K, Biswas M, Samman M M 1991 Damage detection from changes in curvature mode shapes. J. Sound Vibr. 145: 312-332

Raghuveer M R, Boppardikar A S 2001 Wavelet transforms - Introduction to theory and applications (Pearson Education Asia)

Rajagopalan N, Lakshmanan N, Muthumani K 1996 Stiffness degradation of reinforced concrete beams under repeated low energy impact loading. Indian Concrete J. 69: 227-234 
Rajagopalan N, Lakshmanan N, Jeyasekar C A 1999 Damage assessment in reinforced concrete beams using natural frequencies. J. Struct. Eng. India 26: 165-172

Ratcliffe C P 2000 A frequency and curvature based experimental method for Locating damage in structures. Trans. ASME 122: 324-329

Sheinman I 1996 Damage detection and updating of stiffness and mass matrices using mode data. Comput. Struct. 59: 149-156 